University of Nebraska - Lincoln

DigitalCommons@University of Nebraska - Lincoln

Uniformed Services University of the Health

Sciences

U.S. Department of Defense

2011

\title{
Positive emotional style and subjective, cardiovascular and cortisol responses to acute laboratory stress
}

\author{
Sophie Bostock \\ University College London \\ Mark Hamer \\ University College London \\ Andrew J. Wawrzyniak \\ Uniformed Services University \\ Ellen S. Mitchell \\ Unilever Research and Development \\ Andrew Steptoe \\ University College London, a.steptoe@ucl.ac.uk
}

Follow this and additional works at: https://digitalcommons.unl.edu/usuhs

Part of the Medicine and Health Sciences Commons

Bostock, Sophie; Hamer, Mark; Wawrzyniak, Andrew J.; Mitchell, Ellen S.; and Steptoe, Andrew, "Positive emotional style and subjective, cardiovascular and cortisol responses to acute laboratory stress" (2011). Uniformed Services University of the Health Sciences. 84.

https://digitalcommons.unl.edu/usuhs/84

This Article is brought to you for free and open access by the U.S. Department of Defense at DigitalCommons@University of Nebraska - Lincoln. It has been accepted for inclusion in Uniformed Services University of the Health Sciences by an authorized administrator of DigitalCommons@University of Nebraska Lincoln. 


\title{
Positive emotional style and subjective, cardiovascular and cortisol responses to acute laboratory stress
}

\author{
Sophie Bostock ${ }^{\mathrm{a}}$, Mark Hamer ${ }^{\mathrm{a}}$, Andrew J. Wawrzyniak ${ }^{\mathrm{b}}$, \\ Ellen S. Mitchell ${ }^{c}$, Andrew Steptoe ${ }^{a, *}$
}

\author{
a Department of Epidemiology and Public Health, 1-19 Torrington Place, University College London, London WC1E 6BT, UK \\ ${ }^{\mathrm{b}}$ Department of Medical and Clinical Psychology, Uniformed Services University, Bethesda, MD, USA \\ ' Unilever Research and Development, Olivier van Noortlaan 120, 3133 AT, Vlaardingen, The Netherlands
}

Received 9 October 2010; received in revised form 13 February 2011; accepted 14 February 2011

KEYWORDS
Positive emotional style;
Positive affect;
Blood pressure;
Cortisol;
Mental stress;
Recovery

\footnotetext{
* Corresponding author at: Psychobiology Group, Department of Epidemiology and Public Health, University College London, British Heart Foundation, 1-19 Torrington Place, London WC1E 6BT, UK. Tel.: +44 207679 1804; fax: +44 2079168542.

E-mail address: a.steptoe@ucl.ac.uk (A. Steptoe).
}

Summary The relationships between positive emotional style and acute salivary cortisol and cardiovascular responses to laboratory stress tasks were examined in 40 young women (mean age $=28.8$ years). Positive emotional style $(\mathrm{PES})$ was measured by aggregating daily positive mood rating scales over one week. Negative affect was assessed with the short form Profile of Mood States. Salivary cortisol was measured in response to two behavioural tasks, a 5 min speech task and a 5 min mirror tracing task. Blood pressure (BP) and heart rate responses were monitored using a Finometer during baseline, tasks and recovery. Higher PES was associated with more complete diastolic BP recovery $(p=0.027)$ and lower acute cortisol response to stress $(p=0.018)$, after adjusting for baseline measures, age, BMI and negative affect. Individuals with higher PES reported lower subjective tension during the tasks and perceived the tasks as more controllable. There were no differences in ratings of task involvement or in objective measures of task performance. A retrospective measure of positive affect (POMS vigour) was associated with diastolic BP recovery but not cortisol responses or subjective tension. The findings suggest that positive affective traits, assessed using repeated assessments of daily mood, are related to adaptive recovery from acute psychological stress. Our results reinforce evidence linking positive affect with adaptive diastolic BP recovery, while extending the results to cortisol. Investigations into the biological correlates of affective traits should consider utilising repeated measures of experienced affect.

(C) 2011 Elsevier Ltd. All rights reserved.

\section{Introduction}

Positive affect can be defined as the feelings that reflect a state of pleasurable engagement with the environment such as happiness, joy, excitement and contentment (Tomkins, 1963). There is growing evidence that positive affect and 
positive psychological well-being are associated with favourable health outcomes and longevity (Ostir et al., 2001; Kubzansky and Thurston, 2007; Chida and Steptoe, 2008). For example, Davidson et al. (2010) recently showed that the expression of positive emotions, assessed by trained nurse interviewers, predicted incident coronary heart disease (CHD) 10 years later in a sample of 1739 Canadian men and women (adjusted hazard ratio, 0.78; 95\% C.I. 0.63$0.96)$. The prospective association between positive affect and CHD was not reliant on self-report measures of affect and remained robust after adjusting for age, sex and cardiovascular risk factors, including depression and hostility. These associations are corroborated by other longitudinal prospective studies that have controlled for important covariates and biological risk factors (Shirai et al., 2009; Tindle et al., 2009).

Both behavioural and psychobiological processes are thought to underlie the association between positive affect and health (Pressman and Cohen, 2005; Steptoe et al., 2009). For example, not smoking and taking regular physical exercise were consistently related to psychological well-being in a study of 17,000 young adults across 21 countries (Grant et al., 2009). However, in a meta-analysis of studies of positive affect and mortality, statistical adjustment for behavioural factors did not compromise the protective effects of positive affect (Chida and Steptoe, 2008). Psychobiological pathways linking positive emotions and health outcomes are not yet well understood but may include variations in the activation and/or control of neuroendocrine, autonomic, immune and inflammatory pathways. Positive emotional dispositions have been associated with enhanced parasympathetic nervous system control in CHD patients (Bhattacharyya et al., 2008), increased antibody response to a vaccine (Marsland et al., 2006), and reduced daily cortisol output (Polk et al., 2005; Steptoe et al., 2008). Positive dispositions could influence cognitive appraisal of potentially 'stressful' daily events, leading to lower threat perceptions and reduced activation of the autonomic nervous system and hypothalamic-pituitary (HPA) axis (Lazarus and Folkman, 1984). Positive affect may also be linked to the sensitivity of central stress control mechanisms without cognitive awareness. Both positive emotional states and traits have been associated with left-sided activation in anterior brain regions (Davidson, 2004). These areas have a close association with the regulation of cardiovascular and neuroendocrine systems (Critchley, 2005).

In this study, we investigated whether trait positive affect was associated with task appraisals, physiological blood pressure and cortisol in response to two laboratory stress tasks. Psychophysiological stress testing allows individual differences in responses to standardised behavioural challenges to be evaluated and related to psychosocial factors under controlled conditions (Chida and Hamer, 2008). Tugade and Fredrickson (2004) carried out experiments involving student participants which demonstrated that positive affect was associated with more rapid recovery of cardiovascular measures to baseline levels following stress. Cognitive appraisal of the task as 'less threatening' mediated faster recovery. According to Fredrickson's (2001) broaden-andbuild theory, negative emotions narrow an individual's thought-action repertoire and prepare the body for action, while positive emotions have broadening effects which can promote physiological recovery from negative emotional arousal. Papousek et al. (2010) recently found showed that trait but not state positive affect was associated with more complete cardiovascular and subjective post-stress recovery from an academic stressor.

We found supporting evidence for an association between positive affect and accelerated BP recovery in an earlier study (Steptoe et al., 2007). Aggregated ecological momentary assessments (EMA) of happiness assessed over two days were associated with more rapid diastolic BP recovery and lower absolute systolic BP levels before and after stress, even after controlling for negative affect. In contrast, a recent study of over 300 adults reported an association between positive affect and BP stress reactivity but not recovery in response to emotional recall tasks (Brummett et al., 2009). Both BP reactivity and delayed recovery have been related to increased CHD risk prospectively (Chida and Steptoe, 2010). The first aim of the study was to see if we could replicate the association between positive affect and BP recovery to mental stress tasks. Following the broaden-and-build theory, we hypothesised that individuals disposed to positive affect would perceive the tasks to be less stressful and recover more quickly.

In addition to cardiovascular outcomes, we also assessed cortisol stress responses. Evidence from naturalistic studies indicates that positive affect over several days is inversely related to daily cortisol output, independent of negative affect (Polk et al., 2005; Steptoe et al., 2005, 2008). However, findings are not entirely consistent; in a study measuring cortisol and current mood amongst 556 women over five days, Jacobs et al. (2007) found that only negative affect was independently associated with cortisol. Participants in naturalistic studies may be differentially exposed to daily stressors, which may affect transient mood states and cortisol release (Smyth et al., 1998). Individual differences in cortisol's diurnal rhythm may make it difficult to isolate the relationship between positive affect traits and cortisol reactivity to stress. To our knowledge the relationship between positive affective traits and acute salivary cortisol stress responses under controlled conditions has not been reported. Laboratory stress tasks that are perceived to be uncontrollable or characterised by social-evaluative threat have been shown to increase cortisol release (Dickerson and Kemeny, 2004). The second aim of our study was to investigate whether positive affect was associated with cortisol response to acute laboratory stress. We hypothesised that individuals with greater positive emotional dispositions would perceive the tasks to be less stressful (more controllable and less threatening), and have a lower cortisol response, after adjusting statistically for negative affect. To limit variability in cortisol responses in this small study we recruited only women. Psychophysiological stress responses tend to be greater in individuals experiencing a higher background level of stress (Chida and Hamer, 2008) so we recruited individuals with elevated scores on the 12-item General Health Questionnaire (GHQ12) (Goldberg et al., 1997), a measure of psychological distress, but excluded any individuals suffering from anxiety or depressive disorders.

An important issue in studying the psychophysiological correlates of positive affect is the method of measuring positive psychological states. We previously found that positive affect assessed retrospectively over the past week had weaker associations with biological stress responses than 
EMA-derived happiness (Steptoe et al., 2007). One possible explanation is that measures aggregated over several occasions during the day or across days provide more reliable estimates of typical affective traits, since they may be less prone to recall bias, brief peak moods and the dominance of current affect (Kahneman et al., 2006). Alternatively, the difference may reflect the distinction between experienced and recollected affect or the 'memory-experience gap' (Miron-Shatz et al., 2009). Unfortunately, EMA involves a relatively heavy participant burden, which may limit its suitability for certain populations. The third aim of this study was to trial a once-a-day mood rating scale completed for one week to provide an aggregate measure of positive affect. We collected affect ratings every evening over seven days through the Internet, and computed aggregated mean daily positive affect ratings (positive emotional style, PES). We hypothesised that PES over 7 days would correlate more closely with biological stress responses than a retrospective measure of positive affect (the Profile of Mood States mental vigour scale) completed at the time of stress tests. To establish whether associations between positive affect and biology were independent of negative affect, we adjusted for two alternative measures of negative affect: the negative items from the POMS and depressive symptoms measured via with the Centre for Epidemiological Studies Depression Scale (CES-D).

\section{Method}

\section{Participants}

Forty women aged $21-45$ years were recruited from the staff and student population of University College London (UCL). All were non-smokers, consumed fewer than 14 units of alcohol per week and reported being in good health at the time of the study. Women taking prescription medications, excluding contraception, were excluded from the study. All participants completed an online version of GHQ12 and those prone to stress, scoring $>2$, were eligible (Goldberg et al., 1997). Participants gave written consent to participate in the study and ethical approval was obtained from UCL Ethics Committee. Participants were given an honorarium of $£ 30$ on completion. Participants were recruited as part of a larger study to examine the effects of oral GABA (gamma-aminobutyric acid) on psychophysiological stress responses on 70 women.

\section{Procedure}

Eligible participants attended a face to face meeting at which they were instructed to record their mood using an online questionnaire at the end of the day for seven days preceding the laboratory stress session. On the day of testing, participants were instructed not to have drunk caffeinated beverages or eaten large meals at least $2 \mathrm{~h}$ before the study and not to have consumed alcohol or exercised on the previous evening. Participants who reported colds or other infections on the day of testing were re-scheduled. Testing was completed individually in a light and temperature-controlled laboratory between 12:00 h and 17:00 h. Physical measurements including height, weight, percentage body fat, waist and hip circumference were made using standardised methods, and body mass index (BMI) was calculated. Participants rinsed their mouths to avoid contamination of saliva samples. Blood pressure and heart rate (HR) were monitored continuously from the finger using a Finometer (TNO Biomedical Instrumentation, Amsterdam, Netherlands) which uses the vascular unloading technique.

Participants completed several psychosocial questionnaires at rest. At 20-min they were randomised to receive either $200 \mathrm{mg}$ oral GABA or placebo in water. GABA had no impact on subjective or objective stress responses so is not discussed further in this article.

After a further 45-min rest period spent sitting quietly, 5 min 'baseline' blood pressure and HR readings were taken followed by a saliva sample. Cardiovascular readings continued during two 5 min standardised behavioural challenges in random order: a mirror tracing task and a socially evaluating speech task, which have been described previously (Hamer et al., 2006). In the speech task participants were given a hypothetical scenario in which they had to verbally defend themselves against the threat of unemployment. They were given 2 min to prepare and then asked to speak into a video camera for $2 \mathrm{~min}$. Participants were told that the tape would later be replayed by the research team and evaluated. The mirror tracing task involved making as few mistakes as possible while tracing a star which could only be seen in a mirror image (Lafayette Instruments, Lafayette, IN, USA). Participants were told that the numbers of mistakes and completed tracings would be recorded. Each task was immediately followed by task ratings of perceived stress, difficulty, control, performance and involvement. Saliva was collected after completion of the second task. After 20 min spent reading quietly, 5 min cardiovascular 'recovery' readings and a final saliva sample were taken. Subjective ratings of stress (tension) were also recorded at baseline, post-tasks and recovery.

\section{Measures}

Positive emotional style (PES) was assessed using an online daily mood rating scale completed every evening for seven days. The scale was an abridged version of questionnaire used previously to link PES to reduced vulnerability to the cold virus (Cohen et al., 2003), higher antibody responses to a vaccine (Marsland et al., 2006), and reduced daily cortisol output (Polk et al., 2005). Participants were asked to rate the degree to which they felt a number of emotions that day on a 5-point Likert scale, ranging from 0 (you have not felt this at all today) to 4 (you felt this way a lot today). PES was defined as the mean daily score for positive emotions comprising subscales of well-being (happy, cheerful); vigour (lively, full of pep) and calm (calm, at ease). Multiple entries on a single day or morning entries were excluded. All participants completed a minimum of 5 valid daily questionnaires (mean 6.9). Internal consistency based on Cronbach's $\alpha$ for PES was 0.89 in this study.

Task impact ratings of perceived difficulty, involvement, performance, controllability were obtained after each task on 7-point Likert scale, from $1=$ not at all to 7 = very. Subjective ratings of current stress (tension) were also scored on 7-point scales.

Ambient mood over the last couple of days was assessed before the laboratory tasks using a short form of the POMS, as 
described previously (Steptoe et al., 1989). Each item was rated from 0 to 4 , with higher scores indicating more intense mood. Six positive items contributed to the mental vigour scale $(\alpha=0.85)$, while 30 negative items made up the measure of negative affect $(\alpha=0.94)$. Scores for both POMS vigour and POMS negative affect could range from 0 to 24 . Depressive symptoms in the past week were assessed with the Centre for Epidemiological Studies Depression Scale (CES-D) (Cronbach $\alpha=0.83$ ) (Radloff, 1977).

Saliva samples were collected using a passive drool technique. Free salivary cortisol has been shown to correlate highly with blood cortisol (Kirschbaum and Hellhammer, 1994). Saliva was decanted into standard centrifugation tubes and frozen immediately $\left(-80^{\circ} \mathrm{C}\right)$. On completion of the study sessions, salivary cortisol was measured via enzyme-linked immunosorbent assay (ELISA) (SLV-2930, DRG International, Inc., USA) at Technical University Dresden, Germany. The intra- and inter-assay coefficients of variation were less than $8 \%$.

\section{Statistical analysis}

Forty volunteers completed the daily mood questionnaires and subjective ratings during the laboratory stress session. Complete cortisol data were available for 35 participants and complete blood pressure data were available for 31 participants owing to equipment faults. Blood pressure (BP) and heart rate (HR) were averaged into baseline, speech task, mirror task and recovery trials. Blood pressure recovery was scored as the difference between levels during the 20 25 min post-task recovery trial and baseline; more positive scores therefore indicate delayed (incomplete) recovery. Reactivity was calculated by subtracting the baseline values from the mean values measured during the mirror and speech tasks. Cortisol release at baseline, post-tasks and recovery were transformed using natural logarithms to improve the fit to the normal distribution, but raw values are presented in the tables and figures.

Mean PES approximated a normal distribution. Bivariate Pearson correlations were used to examine the associations between PES and normally distributed baseline characteristics. To illustrate the differences between subjects with higher and lower PES, subjects were divided at the median value to compare high and low PES groups. Mean baseline characteristics were compared using $t$-tests. The coefficient of variation in PES was also calculated.

Overall patterns of blood pressure, heart rate, cortisol release (logarithmic transformation) and subjective stress response over the time course of the experiment were analysed with repeated measures analysis of variance with PES group (high, low) as the between person factor and trial (baseline, tasks, recovery) as the within-subject factor. The Greenhouse Geisser correction for degrees of freedom was applied where appropriate. In accordance with the contrast analysis approach advocated by Rosenthal and Rosnow (1985), focused linear regression analyses were then used to examine the relationship between PES and task ratings, cortisol release, cardiovascular stress reactivity and recovery at the different trials. In addition, supplementary table details correlations between raw PES scores and the other variables included in this study. Reactivity and recovery regression models were adjusted for baseline levels of the relevant measure. Covariates also included age and $\mathrm{BMI}$ which are established predictors of basal blood pressure and cardiovascular stress reactivity (Matthews and Stoney, 1988; Steptoe and Wardle, 2005a). To control for negative affect, the negative affective items from the POMS were subsequently added as a covariate to each model. These analyses were repeated by replacing POMS negative affect with CES-D to ensure that depressive symptoms were controlled for. Repeated measures analyses of variance and regression analyses were repeated using the POMS mental vigour scale as the between-person factor. Regression results are presented as unstandardised $(B)$ regression coefficients with $95 \%$ confidence intervals. Mean values are presented \pm standard deviations. All analyses were conducted using SPSS version 16 .

\section{Results}

Seven day aggregate PES ratings ranged from 0.86 to 3.36 . All participants were at risk of medium to high levels of psychological distress, with GHQ-12 scores between 3 and 12 (mean score $7.5 \pm 2.8$ ). Table 1 illustrates the differences between subjects higher and lower in PES, divided at the median. PES was not correlated with age, BMI, waist circumference, resting blood pressure, heart rate, cortisol or self-rated tension. The coefficient of variation in PES across days did not vary with mean PES. The three PES subscales (well-being, vigour and calm) were significantly correlated with one another $(r=0.55-0.70)$. Higher trait PES was strongly correlated with higher POMS vigour scores $(r=0.476, p=0.002)$ inversely correlated with POMS negative affect $(r=-0.485$, $p=0.002)$ and CES-D scores $(r=-0.544, p<0.001)$. POMS vigour was inversely associated with POMS negative affect $(r=-0.397, p=0.011)$.

\section{Subjective tension ratings}

Repeated measures analysis of variance on subjective tension ratings revealed a significant group by trial quadratic effect $(F(1,38)=4.92, p=0.033)$, illustrated in Fig. 1. PES was not associated with baseline or recovery levels of subjective tension, but during the tasks, participants with higher PES experienced lower subjective tension $(B=-0.935, C . I$. -1.683 to $-0.186, p=0.016$ ) after adjustment for baseline stress levels, age, BMI and POMS negative affect (Table 2). Positive emotional style accounted for $11.8 \%$ of the variance in tension during tasks, independently of other factors. Similar results were found when CES-D depressive symptoms replaced POMS negative affect in the model $(B=-1.040, C . I$. -1.809 to $-0.270, p=0.010)$. Individuals with greater PES were therefore less subjectively stressed by tasks, even after negative affect had been taken into account. There were no significant differences in objective measures of mirror task performance (number of tracings or errors) or differences in task involvement with PES. Regression analyses also showed associations between PES and higher task controllability ( $B=1.054$, C.I. 0.230-1.878, $p=0.014)$, perceived task performance $(B=0.669$, C.I. 0.007-1.332, $p=0.048)$ and lower perceived difficulty $(B=-0.677, C . I .-0.010$ to -1.343 , $p=0.047$ ), controlling for age, BMI and POMS negative affect. Associations with task control and perceived difficulty remained significant after adjustment for CES-D. 
Table 1 Baseline characteristics comparing subjects with lower and higher positive emotional style.

\begin{tabular}{|c|c|c|c|}
\hline & \multicolumn{2}{|c|}{ Positive emotional style (PES) } & \multirow[t]{2}{*}{ Correlation with PES, $r(n=40)$} \\
\hline & Low $(n=20)$ & High $(n=20)$ & \\
\hline Age & $28.6 \pm 6.6$ & $29.0 \pm 5.6$ & 0.073 \\
\hline BMI & $23.4 \pm 4.8$ & $22.7 \pm 4.0$ & 0.060 \\
\hline Waist circumference, cm & $74.5 \pm 12.6$ & $74.0 \pm 8.1$ & 0.046 \\
\hline Diastolic BP, $\mathrm{mmHg}^{\mathrm{b}}$ & $74.5 \pm 9.9$ & $69.2 \pm 7.5$ & -0.127 \\
\hline Systolic BP, mmHg ${ }^{\mathrm{b}}$ & $114.5 \pm 13.6$ & $116.3 \pm 14.6$ & 0.248 \\
\hline Heart rate, bpm ${ }^{b}$ & $69.4 \pm 9.9$ & $65.9 \pm 7.9$ & -0.120 \\
\hline Cortisol, $\mathrm{nmol} / \mathrm{l}^{\mathrm{C}}$ & $5.46 \pm 3.4$ & $4.52 \pm 2.1$ & -0.113 \\
\hline Self-rated tension & $1.60 \pm 1.3$ & $1.85 \pm 1.1$ & 0.008 \\
\hline POMS-vigour & $8.6 \pm 4.3$ & $11.7 \pm 3.8^{a}$ & $0.476^{*}$ \\
\hline POMS-negative affect & $8.4 \pm 3.8$ & $5.0 \pm 2.7^{a}$ & $-0.485^{*}$ \\
\hline CES-D & $19.4 \pm 7.4$ & $12.4 \pm 6.6^{\mathrm{a}}$ & $-0.544^{*}$ \\
\hline PES score & $1.66 \pm 0.37$ & $2.52 \pm 0.35$ & 1 \\
\hline PES coefficient of variation & $0.77 \pm 0.42$ & $0.63 \pm 0.22$ & -0.230 \\
\hline
\end{tabular}

All regression analyses for subjective task ratings were repeated based on the subsample for whom cortisol data were available $(n=35)$. The most robust associations were found between PES and task control $(B=0.943$, C.I. $0.130-$ 1.756, $p=0.024)$ and task stress $(B=-0.887, C . I .-1.603$ to $-0.172, p=0.017)$, after adjusting for age, BMI and POMS negative affect. These relationships also remained significant after adjusting for CES-D. POMS vigour was not significantly associated with subjective tension $(B=-0.092$, C.I. -0.186 to $-0.002, p=0.055$ ) or other subjective ratings during the task, or at any other phase of the experiment after adjusting for covariates.

\section{Cardiovascular responses}

Overall, the tasks elicited increases in blood pressure of approximately $20 \%$. Repeated measures analysis of variance confirmed within-subjects main effects of trial for systolic

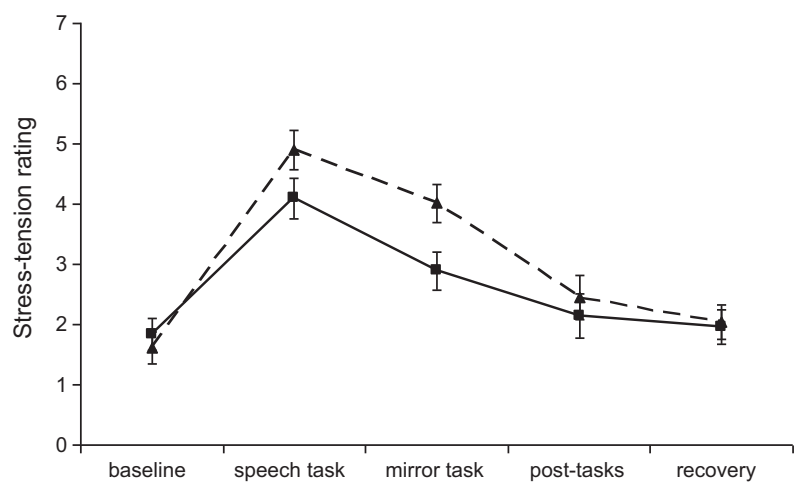

Figure 1 Subjective stress/tension levels for high PES (solid line, squares) and low PES groups (dotted line, triangles) during baseline, speech and mirror tasks and recovery. Error bars represent standard error of mean; adjusted for age and BMI $(n=40)$. and diastolic BP and $\operatorname{HR}(F(3,87)=80.1,114.3$ and 79.4 respectively, $p<0.001)$. Recovery at $20-25$ min was incomplete, with a mean difference between baseline and recovery of $6.9 \mathrm{mmHg}$ for systolic BP (95\% C.I. 4.2-9.6) and $4.0 \mathrm{mmHg}$ for diastolic BP (95\% C.I. 2.5-5.4).

There was a significant group by trial cubic effect in the analysis of diastolic BP $(F(1,29)=4.95, p=0.034$; Fig. 2$)$. Greater PES was associated with more effective diastolic BP recovery, after adjusting for baseline diastolic $\mathrm{BP}$, age, $\mathrm{BMI}$ and negative affect $(B=-3.051$, C.I. -5.270 to -0.382 , $p=0.027$; Table 2). Inclusion of PES in the model independently accounted for $16.3 \%$ of the variance in diastolic BP recovery. This association was maintained after adjusting for depressive symptoms (CES-D) instead of POMS negative affect $(B=-2.689, C . I .-5.365$ to $-0.013, p=0.049)$. There was a similar group by trial cubic interaction for PES and systolic BP $(F(1,29)=4.28, p=0.048)$, but in this case the systolic BP recovery difference was not significant after

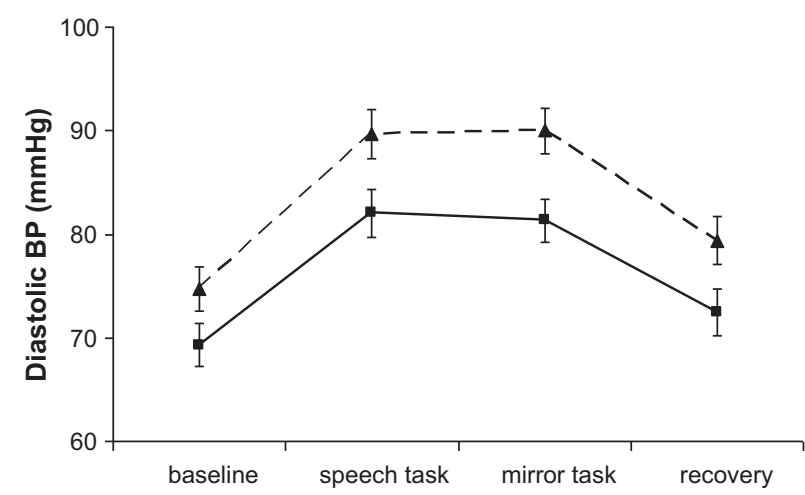

Figure 2 Diastolic BP stress responses for high PES (solid line, squares) and low PES groups (dotted line, triangles) during baseline, speech and mirror tasks and recovery. Error bars represent standard error of mean; adjusted for age and BMI $(n=31)$. 
Table 2 Summary of key multiple linear regression results.

\begin{tabular}{|c|c|c|c|c|}
\hline & $B$ regression coefficient & 95\% C.I. & $t$ & $p$ \\
\hline \multicolumn{5}{|l|}{ Task stress rating } \\
\hline Positive emotional style & $-0.935^{*}$ & -1.683 to -0.186 & -2.54 & 0.016 \\
\hline Baseline stress rating & 0.022 & -0.324 to 0.368 & 0.13 & 0.90 \\
\hline Age & 0.062 & -0.003 to 0.127 & 1.94 & 0.061 \\
\hline BMI & 0.020 & -0.073 to 0.113 & 0.44 & 0.66 \\
\hline POMS negative affect & 0.065 & -0.064 to 0.194 & 1.02 & 0.32 \\
\hline \multicolumn{5}{|l|}{ Diastolic BP recovery ${ }^{\mathrm{b}}$} \\
\hline Positive emotional style & $-3.051^{*}$ & -5.270 to -0.382 & -2.35 & 0.027 \\
\hline Baseline diastolic BP & -0.045 & -0.241 to 0.152 & -0.47 & 0.64 \\
\hline Age & 0.002 & -0.247 to 0.252 & 0.019 & 0.98 \\
\hline BMI & 0.198 & -0.181 to 0.577 & 1.08 & 0.29 \\
\hline POMS negative affect & 0.065 & -0.417 to 0.546 & 0.28 & 0.78 \\
\hline \multicolumn{5}{|l|}{ Cortisol post-task $^{c}$} \\
\hline Positive emotional style & $-0.408^{*}$ & -0.741 to -0.075 & -2.49 & 0.018 \\
\hline Baseline cortisol & $0.845^{*}$ & 0.528 to 1.162 & 5.43 & 0.001 \\
\hline Age & 0.007 & -0.020 to 0.034 & 0.53 & 0.60 \\
\hline BMI & 0.001 & -0.037 to 0.040 & 0.08 & 0.94 \\
\hline POMS negative affect & -0.006 & -0.052 to 0.040 & -0.26 & 0.79 \\
\hline
\end{tabular}

Significant association ( $p \leq 0.05$ ); task stress $n=40,{ }^{b} n=31,{ }^{c} n=35$.

adjusting for covariates $(B=-4.683$, C.I. -9.991 to 0.625 , $p=0.081)$. PES was not related to the magnitude of $B P$ reactivity during the tasks.

POMS vigour showed a comparable association with diastolic BP over time as did PES, with the group by trial cubic effect being significant $(F(1,29)=5.41, p=0.021$. This was again attributable to differences in recovery, with an association between POMS vigour and more effective recovery after controlling for baseline $\mathrm{BP}$, age, gender and negative affect $(B=-0.386$, C.I. -0.739 to $-0.032, p=0.034)$. The relationship with recovery did not persist after adjusting for depressive symptoms $(B=-0.342$, C.I. -0.707 to -0.023 , $p=0.065)$. POMS vigour was not related to systolic BP over time, reactivity or recovery. Neither measure of affect was related to heart rate reactivity or recovery. Task ratings of stress, control or difficulty were not significantly correlated with $\mathrm{BP}$ reactivity or recovery.

\section{Cortisol responses}

Repeated measures analysis of variance confirmed a significant main effect of PES group $(F(1,33)=4.21, p=0.048)$, together with a PES group by time interaction $(F(1,33)=4.98, p=0.033)$. These effects are illustrated in Fig. 3, where it is evident that the high PES group was less responsive than the low PES group. The difference was confirmed in multiple regression on the cortisol value immediately post-tasks, where greater PES was associated with lower cortisol after adjustment for baseline cortisol, age, BMI and negative affect $(B=-0.408, C . I .-0.741$ to -0.075 , $p=0.018$; Table 2). PES accounted for $8.4 \%$ of the variance in post-task cortisol independently of covariates. The association was weakened after adjusting for depressive symptoms but remained close to significance $(B=-0.339$, C.I. -0.687 to $0.008, p=0.055)$. PES was significantly associated with recovery cortisol values in multiple regression, but not after adjusting for negative affect $(B=-0.303$, C.I. -0.754 to $0.147, p=0.179)$. There was no association between POMS vigour and cortisol responses, either through repeated measures analysis of variance or regression on individual task values. For example, post-task cortisol was not associated with POMS vigour after adjusting for baseline cortisol, age, BMI and POMS negative affect $(B=-0.107, C . I .-0.488$ to $0.234, p=0.528$ ).

Of the subjective task ratings, only perceived stress was associated with post-task cortisol values after adjusting for baseline cortisol, age, BMI and negative affect $(B=0.183$, C.I. $0.033-0.334, p=0.018)$.

\section{Discussion}

This study had three objectives: to confirm associations between positive affect and more effective post-stress recovery in diastolic $\mathrm{BP}$, to extend observations to cortisol

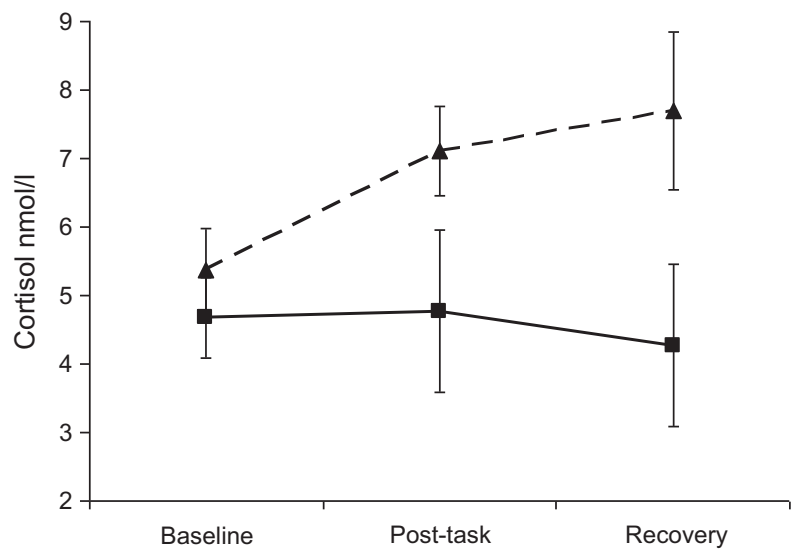

Figure 3 Cortisol release at baseline, after both tasks and at 20-25 min recovery for high PES (solid line, squares) and low PES groups (dotted line, triangles), adjusted for age and BMI. Error bars represent standard error of mean; adjusted for age and BMI $(n=35)$. 
responses, and to investigate differences between experienced affect as assessed through aggregating daily ratings and recollected affect assessed with a standard mood scale. We found that higher positive emotional style (PES) derived from mean daily measures of positive mood was associated with more complete diastolic BP recovery following stress, and that this effect remained after adjusting statistically for age, BMI and negative affect. PES was also associated with lower subjective stress levels and greater perceptions of control during tasks. PES was also associated with reduced cortisol response to stress. Mental vigour assessed using the POMS was associated with diastolic BP recovery but not with subjective task ratings or cortisol responses, indicating a more limited correlation with psychophysiological stress responsivity.

The association between positive emotional style and diastolic BP recovery corroborates the previous finding from our group (Steptoe et al., 2007) in which EMA-derived happiness predicted diastolic BP recovery from laboratory stressors in a sample of young men, independently of negative affect. The effect was substantial, with an additional $16.8 \%$ of variance in diastolic BP recovery being accounted for by positive emotional style. Papousek et al. (2010) measured the cardiovascular stress responses of university students in a scenario designed to simulate a real examination. High levels of trait positive affect (PANAS) were associated with more efficient diastolic but not systolic BP recovery after controlling for negative affect. These studies suggest a relatively consistent diastolic BP recovery effect from acute mental stress with dispositional positive mood in younger adults. In contrast to the current study, psychophysiological stress testing in the Whitehall II cohort of middle-aged men and women revealed no association between EMA-assessed positive affect and BP recovery (Steptoe et al., 2005). The Whitehall II study used a Stroop colour-word interference task rather than a socially evaluative speech task. Brummett et al. (2009) found no association between positive affect and BP recovery after emotional recall tasks, but the recall tasks elicited a relatively weak cardiovascular response (approximately 10\%) and recovery was assessed after only 5 min. Further studies will be required to ascertain whether the nature of the stressor, the age of the participants or recovery time have a critical influence on the presence of an association between positive affectivity and cardiovascular recovery.

Responses to laboratory mental stress tasks have been shown to be relatively stable and reliable over time (Kamarck and Lovallo, 2003). It has been proposed that failure to recover efficiently from acute stress indicates a failure of allostatic regulation (McEwen, 1998). More rapid blood pressure recovery for those high in positive affect may indicate an adaptive advantage in responding to daily life stressors, which over time, could result in lower risk of hypertension (Stewart and France, 2001) and/or accumulated atherosclerotic damage (Steptoe et al., 2006). Although the literature is limited compared with that on cardiovascular reactivity, meta-analysis indicates that delayed post-stress $\mathrm{BP}$ recovery is associated prospectively with increased cardiovascular disease risk (Chida and Steptoe, 2010).

In the current study, individuals higher in PES reported lower subjective stress during the tasks, perceived them to be less difficult, perceived themselves to be in greater control and rated their own performance more highly than in those lower in PES. The most robust associations were with lower perceived stress and greater control. These effects remained after controlling for negative affect, and support a link between positive dispositional affect and more positive cognitive evaluation of stressful tasks (Tugade and Fredrickson, 2004). According to Fredrickson's (2001) broaden-andbuild theory, positive emotions 'undo' the physiological arousal affects of negative emotions. It is notable that there were no differences in ratings of task involvement. This is crucial, since engagement-involvement is a fundamental driver of acute psychophysiological responses (Singer, 1974). The lack of difference indicates that variations in involvement were not responsible for differences between PES groups.

HPA axis reactivity may also link positive affect to cardiovascular recovery and longer term physical health outcomes (Steptoe et al., 2009). Several naturalistic monitoring studies have found an association between positive affect and cortisol output over the day (Polk et al., 2005; Steptoe and Wardle, 2005b). Healthy older adults responding to mental stress tasks with increases in cortisol have been shown to be at significantly higher risk of coronary artery calcification (odds ratio $=2.20$ ) after adjustment for conventional risk factors (Hamer et al., 2010). In a meta-analysis of laboratory studies examining depression and cortisol reactivity to psychosocial stress, Burke et al. (2005) found that depressed patients had higher cortisol levels at 25 min recovery than non-depressed patients. The results of this study indicate that PES is associated with reduced cortisol stress responsivity independently of covariates, demonstrating that the correlations between positive affect and low cortisol identified in naturalistic studies may be extended to investigations of acute stress responsivity under controlled conditions.

It is notable that associations with biology were stronger for the PES than for POMS mental vigour assessed at the beginning of the laboratory session. In a previous experiment, we found that positive affect measured using EMA was associated more consistently with biological responses than was measurement with the PANAS (Steptoe et al., 2007). There are several reasons why this might be the case. One is that repeated measures using the PES over several days may provide a more robust and representative estimate of positive affective traits than a single questionnaire measure. Pressman and Cohen (2005) have argued that measures of affect over multiple time points more reliably identify biological characteristics. Aggregated daily measurements have a lower respondent burden than EMA but may offer similar benefits. A second possibility is that the difference relates to the memory-experience gap articulated by Kahneman and colleagues (Kahneman and Riis, 2005; Miron-Shatz et al., 2009). This suggests that retrospective measures of affect (as assessed with the POMS, PANAS and CES-D) are strongly influenced by salience memory heuristics, affect infusion effects (Forgas, 1995), and other biases, providing less reliable estimates of experienced affect than those derived from repeated measures. Positive emotional style provides a more accurate indication of experienced affect which is likely to be represented in the central nervous system by corticolimbic brain circuitry involving divisions of the cingulate gyrus, the insula and the amygdala (Critchley, 2005). These same areas are responsible for the regulation of cardiovascular and neuroendocrine processes, providing a mechanistic link 
between affective reports and central regulation of the physiological stress responses. Results from the present study suggest that both subcortical regulatory mechanisms and cognitive appraisals may help to explain the link between PES and physiological stress responses; PES was related to self-rated task stress and but measures of task stress were only independently associated with cortisol, not cardiovascular responses.

The correlation between self-rated stress ratings and cortisol in this study is despite findings from a recent meta-analysis showing that only specific cognitive appraisals of acute stressors (such as challenge and novelty) are reliably associated with cortisol response, as opposed to global reports of 'stress' (Denson et al., 2009). It is possible that the fact that our sample were already experiencing a higher than average level of background stress influenced the association between cortisol and subjective task ratings. The current study was limited by relatively basic measures of task appraisal, so we cannot rule out that differences in more subtle cognitive appraisal or adaptive coping mechanisms (undetected by task 'stress' ratings) would help to explain the association between PES and cardiovascular and cortisol stress responses. For example, prolonged negative or ruminative thoughts about a stressor may prolong the physiological response to acute stress (Brosschot and Thayer, 2003).

In this relatively small study we were unable to investigate whether positive emotional style has physiological correlates that are distinct from related dispositions and personality factors. For example, optimism has been linked to lower cortisol responses following a laboratory self-affirmation task (Creswell et al., 2005) while self-esteem has been linked to a lower cardiovascular and inflammatory responses to acute stress (O'Donnell et al., 2008). Further studies could investigate the psychobiological links between the positive emotional style and alternative measures of well-being, particularly in response to interventions to improve psychological measures.

This study was limited to healthy non-smoking young women with some degree of depressive symptoms and may not generalise to other groups. All exceeded the conventional threshold for moderate to severe psychological distress on the GHQ12, and CES-D scores were high, averaging $15.9 \pm 7.8$. It is notable that the mean PES score of the high PES group was only 2.52 on the $0-4$ scale, with the highest individual score being 3.36. Levels of positive affect were not therefore extremely high. This factor may distinguish the present study from other investigations of population samples, avoiding possible ceiling effects in the assessment of positive affect.

We endeavoured to statistically control for the effects of depressive symptoms (CES-D) and negative moods (POMS negative affect) within regression models. However, the limited sample size meant that we were unable to simultaneously adjust for both terms or other potential confounders such as sleep or socioeconomic status, The results are cross-sectional, so causal conclusions cannot be drawn. Stress responses were only measured on a single occasion, but responses to similar tasks have been shown to be reproducible over repeated sessions (Kamarck and Lovallo, 2003). The present results add to the evidence linking positive affect with adaptive diastolic BP recovery following acute stress, while extending these results to cortisol. Aggregated repeated measurements of positive affect had a more robust association with physiological responses than a single retrospective measure. The clinical relevance of these studies would be greatly enhanced by studies of biological responses following interventions to promote and maintain higher positive affect.

\section{Role of funding source}

The funding bodies had no role in the collection, analysis and interpretation of data or the writing of this report.

\section{Conflict of interest}

All authors declare that they have no conflicts of interest.

\section{Acknowledgements}

$\mathrm{SB}, \mathrm{AS}$ and $\mathrm{MH}$ are funded by the British Heart Foundation. This work was partly funded by Unilever Research Vlaardingen. We are grateful to Bev Murray and Lydia Poole for assistance with data collection.

\section{Appendix A. Supplementary data}

Supplementary data associated with this article can be found, in the online version, at doi:10.1016/j.psyneuen. 2011.02.009.

\section{References}

Bhattacharyya, M., Whitehead, D., Rakhit, R., Steptoe, A., 2008. Depressed mood, positive affect, and heart rate variability in patients with suspected coronary artery disease. Psychosom. Med. 70, 1020-1027.

Brosschot, J.F., Thayer, J.F., 2003. Heart rate response is longer after negative emotions than after positive emotions. Int. J. Psychophysiol. 50, 181-187.

Brummett, B.H., Boyle, S.H., Kuhn, C.M., Siegler, I.C., Williams, R.B., 2009. Positive affect is associated with cardiovascular reactivity, norepinephrine level, and morning rise in salivary cortisol. Psychophysiology 46, 862-869.

Burke, H., Davis, M., Otte, C., Mohr, D., 2005. Depression and cortisol responses to psychological stress: a meta-analysis. Psychoneuroendocrinology $30,846-856$.

Chida, Y., Hamer, M., 2008. Chronic psychosocial factors and acute physiological responses to laboratory-induced stress in healthy populations: a quantitative review of 30 years of investigations. Psychol. Bull. 134, 829-885.

Chida, Y., Steptoe, A., 2008. Positive psychological well-being and mortality: a quantitative review of prospective observational studies. Psychosom. Med. 70, 741-756.

Chida, Y., Steptoe, A., 2010. Greater cardiovascular responses to laboratory mental stress are associated with poor subsequent cardiovascular risk status: a meta-analysis of prospective evidence. Hypertension 55, 1026-1032.

Cohen, S., Doyle, W., Turner, R., Alper, C., Skoner, D., 2003. Emotional style and susceptibility to the common cold. Psychosom. Med. 65, 652-657.

Creswell, J.D., Welch, W.T., Taylor, S.E., Sherman, D.K., Gruenewald, T.L., Mann, T., 2005. Affirmation of personal values buffers neuroendocrine and psychological stress responses. Psychol. Sci. $16,846-851$. 
Critchley, H., 2005. Neural mechanisms of autonomic, affective, and cognitive integration. J. Comp. Neurol. 493, 154-166.

Davidson, R.J., 2004. Well-being and affective style: neural substrates and biobehavioural correlates. Philos. Trans. R. Soc. Lond. B. Biol. Sci. 359 (1449), 1395-1411.

Davidson, K., Mostofsky, E., Whang, W., 2010. Don't worry, be happy: positive affect and reduced 10-year incident coronary heart disease: The Canadian Nova Scotia Health Survey. Eur. Heart J. 31, 1065-1070.

Denson, T.F., Spanovic, S., Miller, N., 2009. Cognitive appraisals and emotions predict cortisol and immune responses: a meta-analysis of acute laboratory social stressors and emotion inductions. Psychol. Bull. 135, 823-853.

Dickerson, S.S., Kemeny, M.E., 2004. Acute stressors and cortisol responses: a theoretical integration and synthesis of laboratory research. Psychol. Bull. 130, 355-391.

Forgas, J.P., 1995. Mood and judgment: the affect infusion model (AIM). Psychol. Bull. 117, 39-66.

Fredrickson, B.L., 2001. The role of positive emotions in positive psychology. The broaden-and-build theory of positive emotions. Am. Psychol. 56, 218-226.

Goldberg, D., Gater, R., Sartorius, N., Ustun, T.B., Piccinelli, M., Gureje, O., Rutter, C., 1997. The validity of two versions of the GHQ in the WHO study of mental illness in general health care. Psychol. Med. 27, 191-197.

Grant, N., Wardle, J., Steptoe, A., 2009. The relationship between life satisfaction and health behavior: a cross-cultural analysis of young adults. Int. J. Behav. Med. 16, 259-268.

Hamer, M., O’Donnell, K., Lahiri, A., Steptoe, A., 2010. Salivary cortisol responses to mental stress are associated with coronary artery calcification in healthy men and women. Eur. Heart J. 31, 424-429.

Hamer, M., Williams, E., Vuonovirta, R., Giacobazzi, P., Gibson, L., Steptoe, A., 2006. The effects of effort-reward imbalance on inflammatory and cardiovascular responses to mental stress. Psychosom. Med. 68, 408-413.

Jacobs, N., Myin-Germeys, I., Derom, C., Delespaul, P., van Os, J., Nicolson, N.A., 2007. A momentary assessment study of the relationship between affective and adrenocortical stress responses in daily life. Biol. Psychol. 74, 60-66.

Kahneman, D., Krueger, A., Schkade, D., Schwarz, N., Stone, A., 2006. Would you be happier if you were richer? A focusing illusion. Science 312, 1908-1910.

Kahneman, D., Riis, J., 2005. Living, and thinking about it: two perspectives on life. In: Huppert, F.A., Baylis, N., Keverne, E.B. (Eds.), The Science of Well-Being. Oxford University Press, Oxford, pp. 285-305.

Kamarck, T., Lovallo, W., 2003. Cardiovascular reactivity to psychological challenge: conceptual and measurement considerations. Psychosom. Med. 65, 9-21.

Kirschbaum, C., Hellhammer, D.H., 1994. Salivary cortisol in psychoneuroendocrine research: recent developments and applications. Psychoneuroendocrinology 19, 313-333.

Kubzansky, L., Thurston, R., 2007. Emotional vitality and incident coronary heart disease: benefits of healthy psychological functioning. Arch. Gen. Psychiat. 64, 1393-1401.

Lazarus, R.S., Folkman, S., 1984. Stress, Appraisal and Coping. Springer, New York.

Marsland, A., Cohen, S., Rabin, B., Manuck, S., 2006. Trait positive affect and antibody response to hepatitis $B$ vaccination. Brain Behav. Immun. 20, 261-269.

Matthews, K.A., Stoney, C.M., 1988. Influences of sex and age on cardiovascular responses during stress. Psychosom. Med. 50, 46-56.

McEwen, B., 1998. Protective and damaging effects of stress mediators. N. Eng. J. Med. 338, 171-179.

Miron-Shatz, T., Stone, A., Kahneman, D., 2009. Memories of yesterday's emotions: does the valence of experience affect the memory-experience gap? Emotion 9, 885-891.
O’Donnell, K., Brydon, L., Wright, C., Steptoe, A., 2008. Self-esteem levels and cardiovascular and inflammatory responses to acute stress. Brain Behav. Immun. 22, 1241-1247.

Ostir, G.V., Markides, K.S., Peek, M.K., Goodwin, J.S., 2001. The association between emotional well-being and the incidence of stroke in older adults. Psychosom. Med. 63, 210-215.

Papousek, I., Nauschnegg, K., Paechter, M., Lackner, H., Goswami, N., Schulter, G.C.., 2010. Trait and state positive affect and cardiovascular recovery from experimental academic stress. Biol. Psychol. 83, 108-115.

Polk, D., Cohen, S., Doyle, W., Skoner, D., Kirschbaum, C., 2005. State and trait affect as predictors of salivary cortisol in healthy adults. Psychoneuroendocrinology 30, 261-272.

Pressman, S., Cohen, S., 2005. Does positive affect influence health? Psychol. Bull. 131, 925-971.

Radloff, L., 1977. The CES-D scale: a self-report depression scale for research in the general population. Appl. Psych. Meas. 1, 385-401.

Rosenthal, R., Rosnow, R.L., 1985. Contrast Analysis: Focused Comparisons in the Analysis of Variance. Cambridge University Press.

Shirai, K., Iso, H., Ohira, T., Ikeda, A., Noda, H., Honjo, K., Inoue, M., Tsugane, S., 2009. Perceived level of life enjoyment and risks of cardiovascular disease incidence and mortality: the Japan Public Health Center-Based Study. Circulation 120, 956-963.

Singer, M.T., 1974. Engagement-involvement: a central phenomenon in psychophysiological research. Psychosom. Med. 36, 1-17.

Smyth, J., Ockenfels, M.C., Porter, L., Kirschbaum, C., Hellhammer, D.H., Stone, A.A., 1998. Stressors and mood measured on a momentary basis are associated with salivary cortisol secretion. Psychoneuroendocrinology 23, 353-370.

Steptoe, A., Edwards, S., Moses, J., Mathews, A., 1989. The effects of exercise training on mood and perceived coping ability in anxious adults from the general population. J. Psychosom. Res. 33, 537-547.

Steptoe, A., Wardle, J., 2005a. Cardiovascular stress responsivity, body mass and abdominal adiposity. Int. J. Obes. 29, 1329-1337.

Steptoe, A., Wardle, J., 2005b. Positive affect and biological function in everyday life. Neurobiol. Aging 26, 108-112.

Steptoe, A., Dockray, S., Wardle, J., 2009. Positive affect and psychobiological processes relevant to health. J. Pers. 77, $1746-1776$.

Steptoe, A., Donald, A.E., O’Donnell, K., Marmot, M., Deanfield, J.E., 2006. Delayed blood pressure recovery after psychological stress is associated with carotid intima-media thickness: Whitehall psychobiology study. Arterioscler. Thromb. Vasc. Biol. 26, 2547-2551.

Steptoe, A., Gibson, L., Hamer, M., Wardle, J., 2007. Neuroendocrine and cardiovascular correlates of positive affect measured by ecological momentary assessment and by questionnaire. Psychoneuroendocrinology 32, 56-64.

Steptoe, A., O'Donnell, K., Badrick, E., Kumari, M., Marmot, M., 2008. Neuroendocrine and inflammatory factors associated with positive affect in healthy men and women: the Whitehall II study. Am. J. Epidemiol. 167, 96-102.

Steptoe, A., Wardle, J., Marmot, M., 2005. Positive affect and health-related neuroendocrine, cardiovascular, and inflammatory processes. Proc. Natl. Acad. Sci. U.S.A. 102, 6508-6512.

Stewart, J.C., France, C.R., 2001. Cardiovascular recovery from stress predicts longitudinal changes in blood pressure. Biol. Psychol. 58, 105-120.

Tindle, H., Chang, Y.F., Kuller, L., Manson, J., Robinson, J., Rosal, M., Siegle, G., Matthews, K., 2009. Optimism, cynical hostility, and incident coronary heart disease and mortality in the Women's Health Initiative. Circulation 120, 656-662.

Tomkins, S.S., 1963. Affect Imagery Consciousness: The Positive Affects. Springer, New York.

Tugade, M., Fredrickson, B., 2004. Resilient individuals use positive emotions to bounce back from negative emotional experiences. J. Pers. Soc. Psychol. 86, 320-333. 